\title{
Clinical study of single-visit root canal treatment with a nickel-titanium (Ni-Ti) rotary instrument combined with different ultrasonic irrigation solutions for elderly patients with chronic apical periodontitis
}

\author{
Zhenyu Tang*, Hui Wang and Shiyong Jiang \\ Department of Stomatology, Guilin Stomatological Hospital, No. 5 Sanduo Road Guilin, 541001, \\ China
}

\begin{abstract}
The study involved 300 elderly patients with chronic periapical periodontitis. The patients were randomly assigned into three groups. The treatment for group A used a Mtwo Ni-Ti rotary instrument combined with ultrasonic irrigation of a $2.5 \% \mathrm{NaOCl}$ solution. The group $\mathrm{B}$ used the same instrument combined with ultrasonic irrigation of an active silver ion antibacterial solution. The group $\mathrm{C}$ used the same instrument combined with syringe irrigation of a $2.5 \% \mathrm{NaOCl}$ solution. The root canal fillings were performed immediately after canal preparation. Twenty-four hours after the procedure, patients self-assessed pain levels according to the VAS table. The three groups returned after seven days so their postoperative acute reactions could be evaluated clinically. After six and twelve months, efficacy was evaluated. The self-assessed pain levels for group A and B were significantly lower than group C. The incidence of postoperative acute reactions after seven days for group A and B were significantly lower than those of group C. The effective rates after six and twelve months did not differ among these groups. The single-visit root canal treatment with a nickel-titanium rotary instrument combined with ultrasonic irrigation for elderly patients with chronic periapical periodontitis achieved short and long term efficacy and stability.
\end{abstract}

Keywords: Chronic apical periodontitis, single-visit root canal treatment, ultrasonic irrigation, active silver ion antibacterial solution

\section{Introduction}

A root canal treatment is a procedure that removes the necrotic and infected material within the root canal and fills the canal after proper disinfection. It removes the negative stimulus on the periapical tissues of the root canal and promotes the healing of periapical lesions. Root preparation, disinfection, and root filling determine the efficacy of the treatment. Due to the length and complexity of the treatment, the need for multiple re-examinations, and the postoperative side effects, some elderly

\footnotetext{
* Address for correspondence: Zhenyu Tang, Department of Stomatology, Guilin Stomatological Hospital, No. 5 Sanduo Road Guilin, 541001, China. Tel.: +86 773 2868457; Fax:+86773 2833704; E-mail: twzy2007@163.com.
} 
Table 1

Patients' information divided by group

\begin{tabular}{llllllllll}
\hline Group & Teeth Number & \multicolumn{3}{c}{ Gender } & \multicolumn{2}{c}{ Teeth location } & & \multicolumn{3}{c}{ PAI Grade } \\
& & Male & Female & Incisor/Canine & Premolars & Molar & & \\
\hline & & & & & & & 3 & 4 & 5 \\
A & 120 & 51 & 49 & 39 & 22 & 59 & 59 & 44 & 17 \\
B & 122 & 50 & 50 & 40 & 27 & 56 & 64 & 40 & 17 \\
C & 118 & 52 & 48 & 35 & 30 & 53 & 58 & 41 & 19 \\
P value & & 0.961 & & 0.765 & & & 0.956 & \\
\hline
\end{tabular}

patients are resistant to the treatment and will delay or even forgo treatment.

After we performed a proper root canal preparation for the chronic periapical periodontitis patients with nickel-titanium rotary instruments, we conducted ultrasonic irrigation with different washing solutions. We used traditional syringe irrigation as the control. We finished the procedure with a single-visit root canal filling and compared the clinical efficacies of different treatments.

\section{Treatment and method}

\subsection{General information}

Our participants were 300 elderly patients who visited our dental office between 2010/02 and 2013/12. X-ray films and clinical symptoms had diagnosed these patients with chronic periapical periodontitis, and they needed root canal treatments. The patients were between 60 to 72 years old, with a mean age of 61.3. The patients' periapical indexes (PAI) were determined according to the bone destruction of the teeth with symptomatic apical periodontitis. We randomly assigned the 300 patients into three groups: A, B, and C, as shown in Table 1. The patients must have exhibited the following criteria: formation of apical foramen, no absorption within the root canal, endodontic treatment had not been performed, no acute pain, and an apical alveolar bone resorption area with a diameter $\leq 5 \mathrm{~mm}$ from the X-ray films. Patients were excluded if they had severe systemic disease, a C-shaped and severe curved root canal, a calcified root canal, severe periodontal disease, a teeth mobility degree $>$ II; a pocket depth $>6 \mathrm{~mm}$; or an inability to return to the office. Group A and B were the test groups, and group $\mathrm{C}$ was the control group, with 100 patients in each group. Group A received a $2.5 \%$ sodium hypochlorite solution in ultrasonic irrigation for root canal preparation; group B received an active silver ion antibacterial solution (Silver \& Health) in ultrasonic irrigation; group C received a $2.5 \%$ sodium hypochlorite solution in traditional syringe irrigation. Among the three groups, there were no significant differences for gender, age, and the distribution of teeth locations $(\mathrm{p}>0.05)$.

\subsection{Method}

\subsubsection{Treatment method}

The diseased pulp tissue was removed by the conventional method. Ten \#K files were used to unblock the area from the root canal to the apical third. The length of the root canal was determined with a root canal length-measuring instrument and the X-ray film of the file inside the canal. The canal preparations were performed differently for each group. According to the modified step-back technique suggested by the company, for group A, a conventional rubber dam was used as "raincoat for teeth"; Glyde gel was used to lubricate the canal, and the Mtwo NiTi files were used for the canal 
preparation. The canal preparation used a multifunctional ultrasound therapy machine with the same model or one size less $\mathrm{K}$ file. The length of the $\mathrm{K}$ file inserted into the canal was $2 \mathrm{~mm}$ less than the working length; the staying time was $60 \mathrm{~s}$, and the file did not touch the canal wall. The ultrasonic irrigation solution was $2.5 \%$ sodium hypochlorite for group A. After ultrasonic irrigation, a soft paper tip was used to dry the canal and AH plus root canal sealer was used to help increase taper for guttapercha with cold lateral condensation filling. Finally, a postoperative X-film was taken to confirm the proper filling.

Group B received active silver ion antibacterial solution in ultrasonic irrigation and the other steps were the same as group A. As the control group, group C received syringe irrigation with $2.5 \%$ sodium hypochlorite solution and a routine root filling. The postoperative X-ray film confirmed that the root filling was at the correct length within the root canal space. All steps were performed by two endodontists at a similar level. An adequate coronal restoration was made after the procedure.

\subsubsection{Call-back and revisit time}

Twenty-four hours after the procedure, the patients received calls to self-assess pain levels. Seven days after the procedure, the patients returned to the dental office, where two endodontists at a similar level performed clinical examinations and recorded the results. At six and twelve months after the procedure, the patients revisited the dental office for X-rays. The endodontists used evaluation criteria to evaluate the treatment based on these X-ray films and the clinical examination results.

\subsection{The evaluation of postoperative reaction}

\subsubsection{The patients' pain self-assessment}

Before the procedure, patients were trained to use the visual analogue scale (VAS) table to perform pain assessment correctly [1]. We used patients' postoperative VAS values to assign them to four grades: grade 0 for a VAS value of 0 for no pain; grade 1 for VAS values from 1 to 3 for mild pain; grade 2 for VAS values from 4 to 6 for moderate pain that is tolerable without analgesics; and grade 3 for VAS values from 7 to 10 for severe pain that is unbearable and requires oral analgesics or emergency treatment.

\subsubsection{The clinical evaluation of postoperative acute reactions}

The evaluations were performed by two endodontists at similar levels [2]. Severe pain indicates spontaneous pain, gum swelling, and moderate-severe percussive pain at the treatment site after the root canal filling. Moderate pain indicates spontaneous pain, no gum swelling, and mild percussive pain at the treatment side. Mild pain indicates no gum swelling or discomfort to percussion at the treatment side. No pain indicates an absence of postoperative pain.

\subsubsection{The clinical evaluations}

With each office revisit, we evaluated the clinical effective rates according to the PAI [3], the clinical examination, and the X-ray film of the lower third of the alveolar bone. The treatment was considered successful if the patient had normal masticatory functions, no discomfort, no percussive pain during the examination, and a PAI of 1 or 2 . The treatment was considered a failure if the patient could not perform normal masticatory functions, experienced discomfort and percussive pain at the examination, and had PAI of 3 or above.

\subsection{Statistics}


Table 2

Comparison the pain self-assessment among the three groups (n, \%)

\begin{tabular}{lllllll}
\hline Group & Total & \multicolumn{2}{l}{ Pain self-assessment } & \multirow{2}{*}{ P value } \\
\cline { 3 - 5 } & & Grade 0 & Grade 1 & Grade 2 & Grade 3 & \\
\hline A & $120(100)$ & $99(82.50)$ & $15(12.50)$ & $4(3.33)$ & $2(1.67)$ & 0.0000 \\
B & $122(100)$ & $103(84.43)$ & $14(11.48)$ & $4(3.28)$ & $1(0.82)$ & \\
C & $118(100)$ & $71(60.17)$ & $26(22.03)$ & $17(14.41)$ & $4(3.39)$ & \\
\hline
\end{tabular}

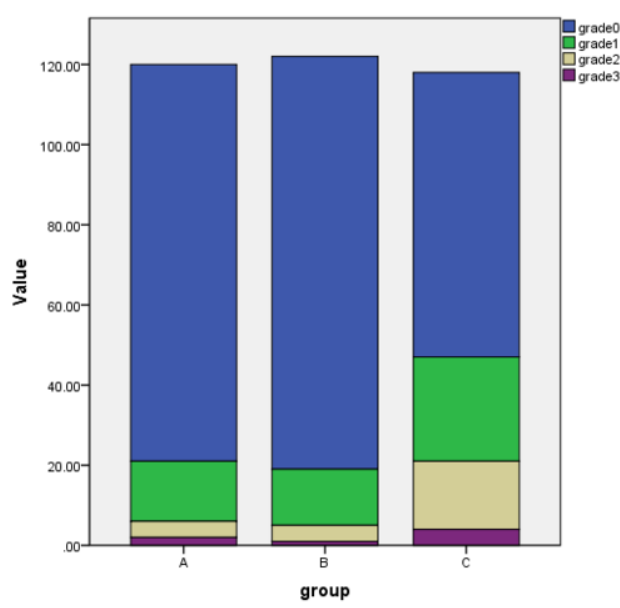

Fig. 1. Four grades of pain self-assessment in three group.

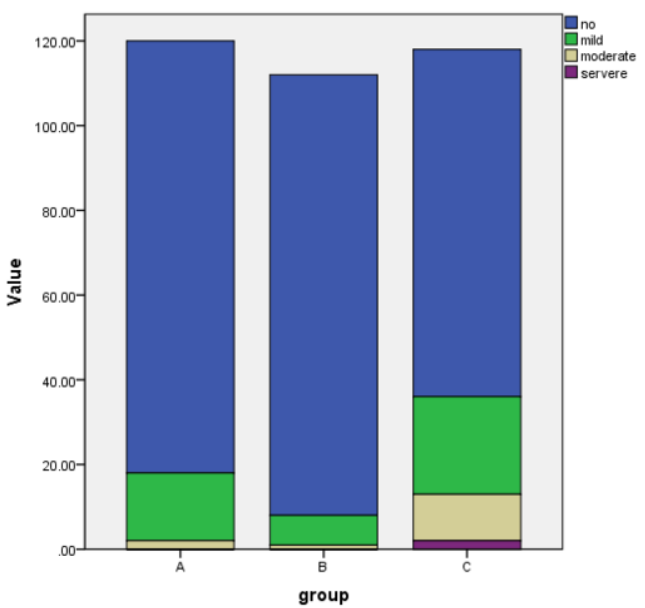

Fig. 2. Four grades of postoperative reactions in three groups.

Kruskal-Wallis $\mathrm{H}$ test was used to compare the degree of pain self-assessment and postoperative reaction among three groups. Multiple comparisons were conducted if the results of Kruskal-Wallis $\mathrm{H}$ test were significant. Chi-square test was used to compare clinical efficacy. SPSS version 18.0 was used to perform the analysis. Differences were considered as statistically significant if $\mathrm{P} \leq 0.05$

\section{Results}

\subsection{Pain level after procedure}

Among the three groups, there is a significant difference in the pain self-assessments $(\mathrm{P}<0.05)$. There is no significant difference in the incidence of moderate to severe pain between group A and $\mathrm{B}$ $(\mathrm{P}=0.778)$. While group $\mathrm{C}$ showed 21 cases of moderate to severe pain at 24 hours after the procedure and the incidence of acute reactions is $17.80 \%$, which is significantly different from group A and B (P $=0.0000)$ (see Table 2 and Figure 1).

\subsection{Postoperative reactions}

The clinical evaluations at the seven days revisit after procedure showed a significant difference in pain distribution among the three groups $(\mathrm{P}=0.0009)$. The difference between group $\mathrm{A}$ and $\mathrm{B}$ was not significant $(\mathrm{P}=0.956)$, while the differences between group $\mathrm{C}$ and group $\mathrm{A}$ or $\mathrm{B}$ were significant $(\mathrm{P}<$ 0.05) (see Table 3 and Figure 2). 
Table 3

Comparison of the postoperative reactions among the three groups (n)

\begin{tabular}{lllllll}
\hline Group & Total & Pain & & & & P value \\
\cline { 3 - 5 } & & No & Mild & Moderate & Severe & \\
\hline A & 120 & 102 & 16 & 2 & 0 & 0.0009 \\
B & 122 & 104 & 7 & 1 & 0 & \\
C & 118 & 82 & 23 & 11 & 2 & \\
\hline
\end{tabular}

Table 4

Comparison of the effective rates among the three groups (n, \%)

\begin{tabular}{llllllll}
\hline Group & A & & B & & C & P value \\
\cline { 1 - 1 } Revisit & Success & Fail & Success & Fail & Success & Fail & \\
6 months & $97(80.83)$ & 23(19.17) & 99(81.15) & 23(18.85) & $88(74.58)$ & $30(25.42)$ & 0.375 \\
12 months & $102(85.0)$ & $18(15.0)$ & $108(88.52)$ & $14(11.48)$ & $92(77.97)$ & $26(22.03)$ & 0.078 \\
\hline
\end{tabular}

\subsection{Evaluation of treatment results}

According to the clinical evaluations, we compared the groups' effective rates at the six months revisit after treatment. The effective rates for group $\mathrm{A}$ and $\mathrm{B}$ are much higher than that of group $\mathrm{C}$ (74.58\%); however, statistically there is no significant difference among the three groups $(\mathrm{P}=0.375)$. Similarly, the effective rates for the three groups do not demonstrate a significant difference at twelve months either $(\mathrm{P}=0.078)$ (see Table 4 and Figures 3 and 4$)$.

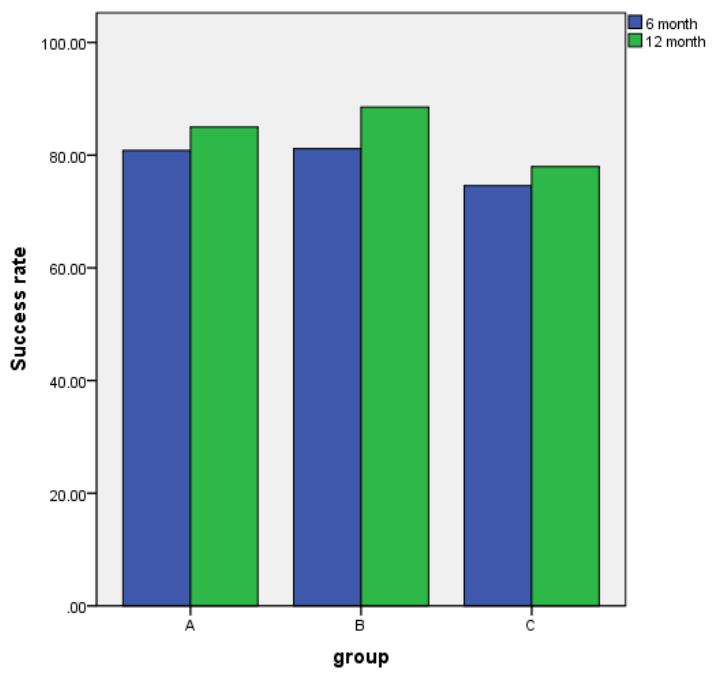

Fig. 3. Success rate at the time point of 6 month and 12 month in three groups.

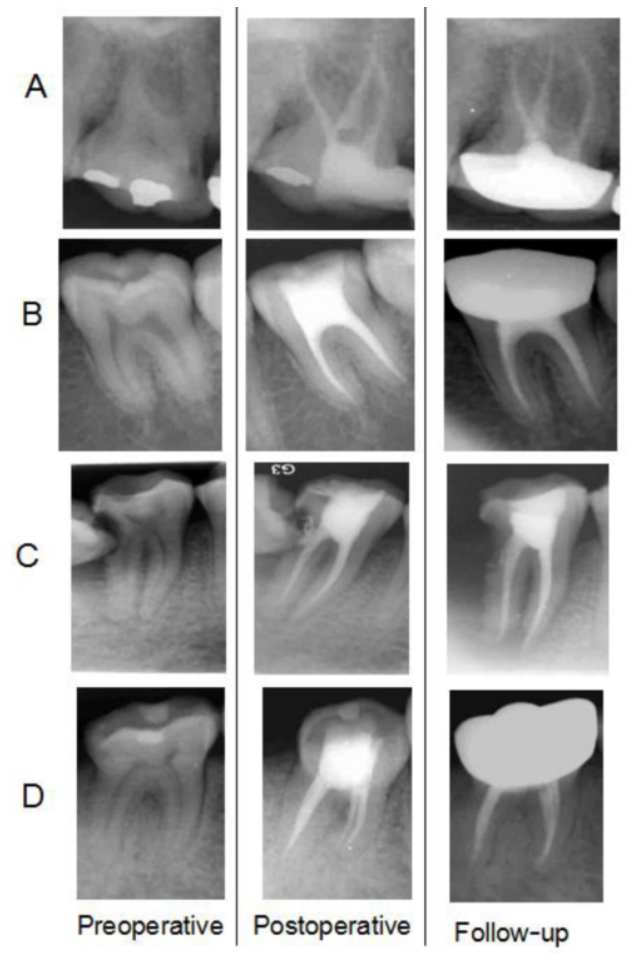

Fig. 4. Preoperative, postoperative, and follow-up radiographs in three groups: A, B healed, C, D failed. 


\section{Discussion}

The fundamental and clinical research on single-visit root canal treatment has been widely applied as early as 19th century [4]. However, the efficacy of these treatments is inconclusive [5]. Some scholars have used the Cochrane method to systematically review literature and have concluded that there is no statistical difference between the efficacies of single-visit and multi-visit root canal treatment [6]. Nevertheless, for elderly patients, the primary criteria for efficacy evaluation lie in a short treatment course, fewer revisits, mild or no pain after treatment, and a high degree of comfort. In recent years, the methods of root canal disinfection have greatly progressed. Except for the use of chemical agents to disinfect the root canal, the use of ozone, or photoactivated disinfection with lowenergy laser, electrochemically activated water, and electric current also have good effects. The study mainly discussed the clinical efficacy of a single-visit root canal treatment with a nickel-titanium rotary instrument combined with ultrasonic irrigation for elderly patients.

Our study first used Mtwo NiTi files to perform proper root canal preparation, then used a 2.5\% sodium hypochlorite solution or an active silver ion antibacterial solution to perform ultrasonic irrigation for group A and B, respectively; we used a $2.5 \%$ sodium hypochlorite solution with traditional syringe irrigation for control group C. Finally, we compared postoperative pain levels, acute reactions, and effective rates. The results demonstrated that the self-assessed pain levels 24 hours after the procedure are significantly lower for group $\mathrm{A}$ and $\mathrm{B}$ than for group $\mathrm{C}(\mathrm{P}<0.05)$. There is no significant difference between group $\mathrm{A}$ and $\mathrm{B}(\mathrm{P}>0.05)$. Group $\mathrm{A}$ and $\mathrm{B}$ also showed lower incidences of postoperative acute reactions. Similarly, at the seven days revisit, clinical examination by the endodontists and the patients' symptoms showed the incidences of postoperative acute reactions for group $\mathrm{A}$ and $\mathrm{B}$ are significantly lower than that of group $\mathrm{C}(\mathrm{P}<0.05)$. The incidences for group $\mathrm{A}$, and $\mathrm{B}$ are similar $(\mathrm{P}>0.05)$.

The patients who suffered from severe pain 24 hours after the procedure were given oral antiinflammatory drugs. At the seven days revisit, the patients in group A and B who took antiinflammatory drugs had no inflammation or acute pain. However, the two patients in group $\mathrm{C}$ who took anti-inflammatory drugs still suffered from severe pain and needed further occlusal adjustments, reopening, and re-treatment of the root canal. The results indicate that compared to the traditional syringe irrigation, Mtwo NiTi files combined with ultrasonic irrigation can enhance the postoperative comfort and reduce acute reactions, thus achieving higher short-term effective rates.

The Mtwo NiTi files from VDW, Germany used in our study are currently the only system that is compatible with conventional root canal preparation. Conventionally, four files are enough for the canal preparation. Compared to the other NiTi system, the procedure is much simpler and easier. The concussion of ultrasonic tips in the root canal can produce the cavitation effect, micro acoustic streaming effect, and synergistic effect [7]. Compared to traditional syringe irrigation, ultrasonic irrigation can enhance the washing solution's ability to dissolve and disinfect the organic matter; remove large amounts of infectious microbial and debris in the smear layer of main root canal, canal collateral, curved canal and/or isthmus [8]; open dentinal tubules; and reduce the chance of leaking debris and fluid mixtures through the apical foramen due to irrigation. Thus, this method is useful in dredging the thinned root canal in elderly patients and can improve the effect of collateral filling. Our study used different solutions to perform ultrasonic irrigation for the patients of group A and B, and found that the self-assessed pain levels and clinical symptoms were all better than those of group $\mathrm{C}$ at the 24 hours and seven days revisit. These results confirm the clinical superiority of ultrasonic irrigation.

The ultrasonic irrigation solutions we used include $2.5 \%$ sodium hypochlorite and an active silver 
ion antibacterial solution. The latter demonstrates no significant difference compared to the more popular $2.5 \%$ sodium hypochlorite solution $(\mathrm{P}>0.05)$. Using patented technology, the active silver ion antibacterial solution changes silver ions into the high valence and high activity $\mathrm{Ag}^{2+}$ and $\mathrm{Ag}^{3+}$. These ions can active absorb bacteria, fungi, and viruses and eliminate these microbes by undermining their active enzymes; they can also reduce inflammation by decreasing the substitution with $\mathrm{Zn}^{2+}$ and $\mathrm{Ca}^{2+}$, and promote epithelial cell regeneration by cooperating with sulfur and nitrogen. Thus as a nonantibiotic fungicide, the active silver ion antibacterial solution is a powerful bactericidal and promotes healing. It can kill most clinical pathogenic microorganisms and has no drug resistance. It is also colorless, odorless, non-irritating, has a $\mathrm{pH}$ of 6-7 that is close to the normal oral $\mathrm{pH}$, and is comfortable to use. No rubber dam is required when using this solution, and it will not corrode the tubes of the ultrasound therapy device. Clinically it can substitute $2.5 \%$ sodium hypochlorite solution in ultrasonic irrigating.

At six and twelve months after the procedure, the effective rates for group $\mathrm{A}$ and $\mathrm{B}$ are higher than group $\mathrm{C}$, but the differences are not statistically significant $(\mathrm{P}>0.05)$. The underlying reason might be that the Mtwo NiTi files' preparation combined with ultrasonic irrigation can perform effective cleaning of the irregular, narrow area inside the canal, apical transport branches, and the turning points of the curved canal. This makes the subsequent root canal filling more perfect and compact. This data confirms that single-visit root canal treatment with NiTi files and ultrasonic irrigation is both reliable and shows long-term efficacy. However, the more long-term clinical efficacy still need further observation.

\section{Conclusions}

This study proved that a single-visit root canal treatment for elderly chronic periapical periodontitis patients with Mtwo NiTi files and ultrasonic irrigation leads to easy postoperative reactions and stable short and long-term efficacies. Under strict control of the indications, this method is promising in clinical application. Furthermore, the active silver ion antibacterial solution we used can replace the $2.5 \%$ sodium hypochlorite as the ultrasonic irrigation solution, as it shows similar results, is odorless, and offers a better patient experience.

\section{Acknowledgments}

This study was supported by the Scientific Research and Technology Development Plan of Guangxi Province (grant No. 14124004-1-10) and the Scientific Research Plan Project of Guilin City (grant No. 20120121-8 and 20140120-8-1). The funders had no role in study design, data collection and analysis, decision to publish, or preparation of the manuscript.

\section{References}

[1] Z.S. Madani, A.A. Moghadamnia, A. Panahi and A. PoorsattarBejeh Mir, Analgesic effect of etoricoxib compared to Ibuprofen on post endodontic pain, Journal of Oral Health and Dental Management 12 (2013), 186-190.

[2] Z.Y. Liu and J.D. Chen, The efficacy of single-visit root canal treatment for elderly patients, Chinese Journal of Geriatric Dentistry 12 (2014), 153-156.

[3] R.A. Jordan, A.L. Holzner, L. Markovic, I. Brueckner and S. Zimmer, Clinical effectiveness of basic root canal 
treatment after 24 months: A randomized controlled trial, Journal of Endodontics 40 (2014), 465-470.

[4] A.C. Xavier, F.C. Martinho,A. Chung, L.D. Oliveira, A.O. Jorge, M.C. Valera and C.A. Carvalho, One-visit versus two-visit root canal treatment: Effectiveness in the removal of endotoxins and cultivable bacteria, Journal of Endodontics 39 (2013), 959-964.

[5] G. Dorasani, K. Madhusudhana and S.K. Chinni, Clinical and radiographic evaluation of single-visit and multi-visit endodontic treatment of teeth with periapical pathology: An in vivo study, Journal of Conservative Dentistry 16 (2013), 484-488.

[6] Y.Y. Sun, X.P. Liang and J. Zhao, The systematic evaluation of the efficacy of single-visit versus multi-visit root canal treatment for chronic pulpitis and periapical periodontitis, Chinese Journal of Geriatric Dentistry 21 (2011), 452-458.

[7] S.M. Andrabi, A. Kumar, A. Zia, H. Iftekhar, S. Alam and S. Siddiqui, Effect of passive ultrasonic irrigation and manual dynamicirrigationon smear layer removal from root canals in a closed apex in vitro model, Journal of Investigative and Clinical Dentistry 5 (2014), 188-193.

[8] F. Ahmetoglu, A. Keles, M. Yalcin and N. Simsek, Effectiveness of different irrigation systems on smear layer removal: A scanning electron microscopic study, European Journal of Dentistry 8 (2014), 53-57. 